\title{
Performance of Several Low-Cost Accelerometers
}

\section{by J. R. Evans, R. M. Allen, A. I. Chung, E. S. Cochran, R. Guy, M. Hellweg, and J. F. Lawrence}

Online Material: Description of typical Class-C MEMS accelerometers; example of Excel analysis sheet; table summaries of box-flip test results, sensor performance, and pricing information.

\section{INTRODUCTION}

Several groups are implementing low-cost host-operated systems of strong-motion accelerographs to support the somewhat divergent needs of seismologists and earthquake engineers. The Advanced National Seismic System Technical Implementation Committee (ANSS TIC, 2002), managed by the U.S. Geological Survey (USGS) in cooperation with other network operators, is exploring the efficacy of such systems if used in ANSS networks. To this end, ANSS convened a working group to explore available Class $\mathrm{C}$ strong-motion accelerometers (defined later), and to consider operational and quality control issues, and the means of annotating, storing, and using such data in ANSS networks. The working group members are largely coincident with our author list, and this report informs instrument-performance matters in the working group's report to ANSS. Present examples of operational networks of such devices are the Community Seismic Network (CSN; csn.caltech.edu), operated by the California Institute of Technology, and Quake-Catcher Network (QCN; Cochran et al., 2009; qcn.stanford.edu; November 2013), jointly operated by Stanford University and the USGS. Several similar efforts are in development at other institutions. The overarching goals of such efforts are to add spatial density to existing Class-A and Class-B (see next paragraph) networks at low cost, and to include many additional people so they become invested in the issues of earthquakes, their measurement, and the damage they cause.

Classes A, B, and C are defined in terms of performance by ANSS (2008). Class A refers to the highest performance, stateof-the-art instrumentation, presently for accelerometers with useful resolution of about 22-24 bits peak-to-peak over \pm 2 to $\pm 4 g$ ranges (sensor roughly US\$2000-4000). Class B is illustrated well by the NetQuakes instrument (GeoSIG model GMS-18) that is an effectively 16-bit (vertical) and 18-bit (horizontal) instrument over $\pm 3 g$ ranges (notwithstanding that longer sample words are recorded; Luetgert et al., 2009,
2010; sensor roughly US\$500-1000). Class C is the lowest performance level potentially usable by ANSS and has useful resolution from about 12 to 16 bits, typically over $\pm 2 g$ ranges (sensor roughly US\$100-200). (E) We describe the design of typical Class- $\mathrm{C}$ accelerometers and provide links on the subject in the electronic supplement to this paper.

In order to facilitate the use of Class- $\mathrm{C}$ sensors in regional networks it is critical that we are able to understand the capabilities and limitations of these instruments. This report describes performance-test results for the following five types of triaxial Class- $\mathrm{C}$ sensors together with their recording systems, public or private:

1. Droid smart phones, one example of a Google Nexus One (we call this, Serial Number 2[SN2]; https://sites.google .com/a/pressatgoogle.com/nexusone/; November 2013), and two examples of HTC Magic phones (we call these SN3 and SN4; there is no SN1; http://www.htc.com/us/; November 2013); note that iPhones, laptop computers, and probably others have similar capability, so we will refer to tested devices generically as smart phones;

2. Gulf Coast Data Concepts (GCDC; gcdataconcepts.com) model X6-2 shipping monitors used to detect drops and bumps of valuable packages during shipment and handling (SNs 4086, 4097, and 4128);

3. JoyWarrior model 24F14 accelerometers ("JWF14"; Code Mercenaries Hard- und Software GmbH, codemercs.com), a type often used to control video games (SNs 1-6; SNs 1,2 , and 5 are $\pm 1 g$ devices, the other three are $\pm 2 g$ devices);

4. two models of similar video-game controllers from O-Navi LLC (o-navi.com), models 23567-A (“O-Navi A") and 23567-B ("O-Navi B"); and

5. two models of Phidgets (phidgets.com), five of model 1056 (SNs 145444-252220), and one prototype model 1043 (SN 999990); these devices are aimed at general prototyping (often for navigation) and amateur users.

Unless otherwise stated, these are $\pm 2 g$ devices. The tests performed were

1. "box flip" tests for $0 \mathrm{~Hz}$ sensitivity, offset, and axis orientations;

2. transfer function tests (response functions) in this case for amplitude, but not phase; 
3. tests of clipping behavior and sensor linearity (sensitivity versus input acceleration level);

4. sensor self-noise levels, which determine useful operating ranges in decibels or bits; and

5. a double integration test to determine whether permanent displacements can be recovered accurately from these accelerometers.

We note a terminology issue between commonly used Class- $\mathrm{A}$ and Class- $\mathrm{B}$ sensors and the Class- $\mathrm{C}$ sensor used here. Although we use essentially the same ANSS tests as for Classes A and $\mathrm{B}$, the outputs of such devices are in volts, with filtering and conversion to counts performed by high-precision recorders. We recommend using a similar set of tests for Class- $\mathrm{C}$ devices, but in those we test here and most others, the analog-to-digital converter (ADC) and likely anti-alias filters are contained within the sensor package, with that package outputting a digital stream of converted data. Thus, we will use the term sensor here to mean those integrated, digital-out packages. Similarly, while the recording methods we used are not complete data acquisition units (DAUs in ANSS parlance) in the sense of combining ADC, timing, storage, and communications, they perform most of those duties, and we therefore call them recorders. (E) Most of these are laptop software supplied by the vendors or network operators (enumerated in the electronic supplement). GCDC devices record ADC counts internally and download them as such, so are complete data acquisition systems, or "DASs" in ANSS parlance. Finally, we did not have direct access to the sensor digital outputs, so what is reported here is muddied by using existing recorders and their software or firmware as provided by the various network operators or sensor vendors. Here, we must assume that the recorders faithfully report sensor outputs in units specific to that model. The most likely exceptions are additional filtering that may be performed by the recorders and filtering, which is performed in our analyses by resampling to 200 samples/s. MATLAB uses a linear-phase finite impulse response [FIR] filter with Kaiser window when downsampling is needed, so these filter effects are likely to be at high frequencies and have little effect on our results.

Tests were performed largely at the USGS Albuquerque Seismological Laboratory (ASL; e.g., Hutt et al., 2011) in June 2012 to evaluate sensitivity, axis orientation, system noise, response functions, clipping and linearity, and the ability to double integrate the acceleration records to recover $400 \mathrm{~mm}$ quasi-static steps in displacement. They were attached to an aluminum plate with removable adhesives because many lack bolt-down provisions; the plate was bolted or clamped to the shake tables. Some of the noise data came from other locations and serial numbers, with the sensor plate simply resting on a concrete pier. These tests are all routinely applied at ASL to Class-A and Class-B systems as well. The Class- $\mathrm{C}$ devices we tested vary in overall performance from poor by seismological standards (smart phones with internal accelerometers; an iPhone we tested a few years prior to the present tests had resolution similar to the present examples) to quite good by the same standards (Phidgets and O-Navi), with the range of performance between those extremes. Note that the smart phones we tested were old models. We hear that newer models perform far better in resolution and noise so our tests may not be representative (R. Allen, personal comm., 2013).

The best devices appear to be have greater resolution than the venerable Kinemetrics SMA-1 optical accelerograph (data from which drove the early decades of earthquake building code development), so we expect they can contribute useable data for some purposes, such as input to ShakeMap and ground-motion prediction equations, if other issues are found tractable. Thus, we believe they are viable candidate instruments for use by host-operated low-cost networks for seismological research, engineering research and practice, and emergency response.

\section{BOX-FLIP TESTS}

Accelerometers of most types have the helpful behavior of a flat response all the way to $0 \mathrm{~Hz}$ representing sensor output when it is motionless. Thus, one can measure basic performance information simply by attaching the sensors rigidly inside an accurately rectilinear box, with the sensor-case orientation well aligned to the box edges. By sequentially placing the box and sensors in each of its six possible orientations on a flat, stable, carefully leveled surface (Fig. 1), one can obtain the static sensitivity, offset, and orientations of every axis;
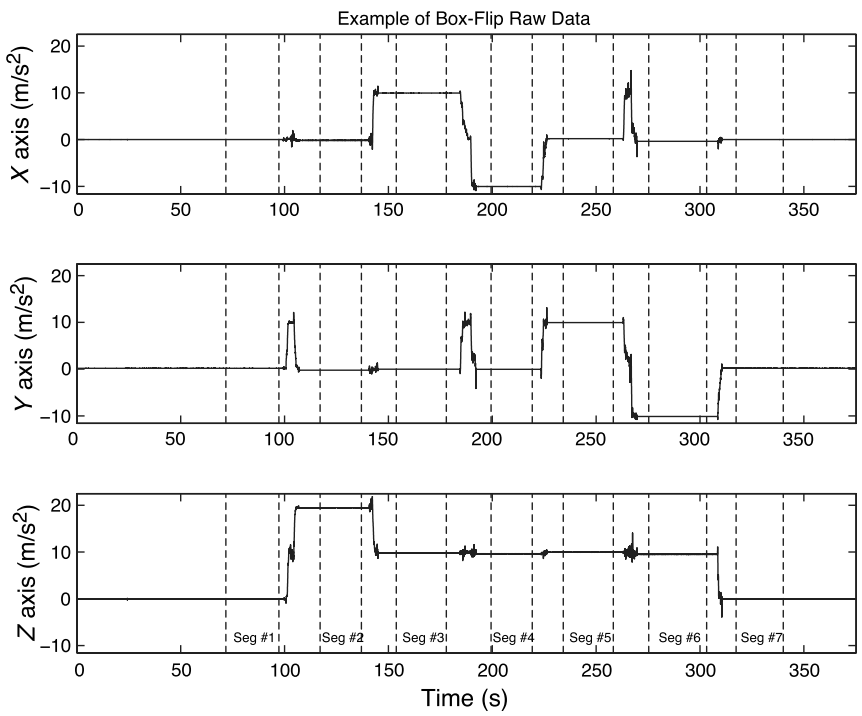

A Figure 1. Example of the raw data for a box-flip test, showing periods of stasis at the six possible orientations of the box at which the sensors are attached and aligned; the first orientation, normal installation orientation, is repeated at the end of the test to constrain instrument drift. Signals between stasis intervals are due to moving the box to its new orientations. The dashed lines indicate the segments for which mean output levels were computed in this example; segments are selected by an analyst. $Z$ axis is offset by $1 g$ because its output is corrected for the Earth's static field, perhaps by simply adjusting the output voltage in the sensor; the correction does not appear to limit sensor range nor to be anything but a simple constant. 
(a)

"X, Phidget 1056, SN 145667"
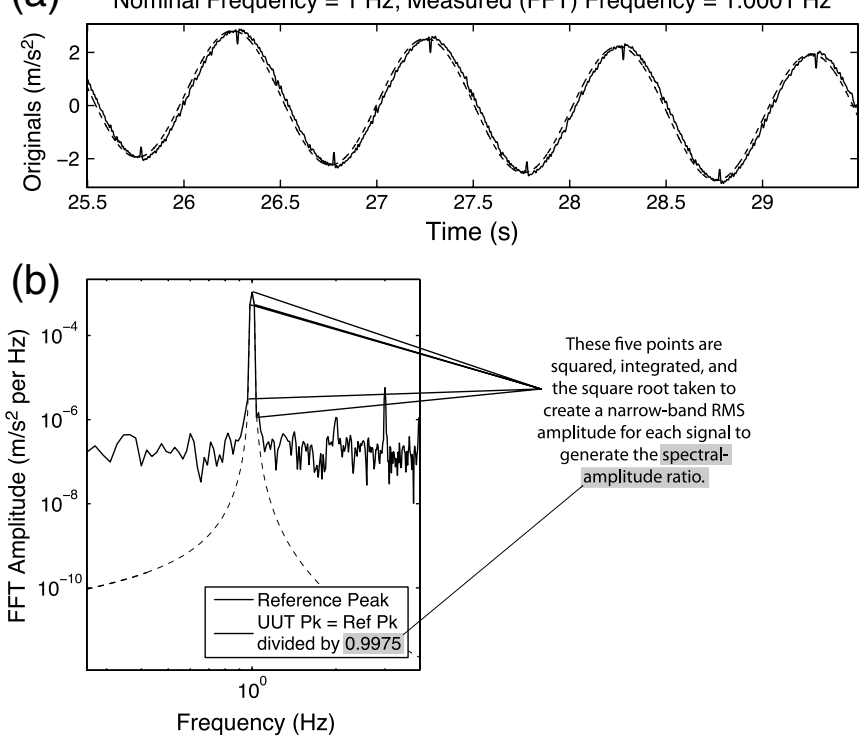

A Figure 2. (a) Example of amplitude-response analysis for a sine-signal input by a shake table (dashed trace, here synthetic) to the output signal (solid). Only the first few cycles of the sine segment are shown, but the entire signal is used for analysis. (b) A narrowband part of the spectral responses (simple fast Fourier transforms [FFTs]) centered at the input sine frequency. The middle five frequency bins (indicated by leaders) are squared and integrated to yield band-limited power in units of acceleration squared); finally, the root is taken to compute accelerationamplitude ratios. The numerical spectral amplitude ratio is shown in that panel as well; the records in (a) have been scaled accordingly.

orientations are relative to the sensor case axes (the latter by comparing outputs due to gravity in each of the $g$-normal orientations that are highly sensitive to deviations from horizontal). Finally, returning the box to its original upright orientation allows a rough estimate of sensor drift over time. Class A and Class B accelerometers are expected to be within $1 \%$ of their expected sensitivities (ANSS, 2008), to have modest inherent offsets so that sensor clipping remains approximately symmetrical about their resting output levels and do not clip asymmetrically, and to have true active sensitivity axes within $1^{\circ}$ of the case axes, which are used during deployment to orient the sensors to the Earth or to buildings, bridges, and other structures of interest. In this instance, because we do not have access to the raw outputs of these MicroElectricalMecanical Systems (MEMS) accelerometers, we use the continuous time series as recorded by supporting software, and then measure the mean output levels of appropriate segments of those data (e.g., Figs. 1 and 2).

\section{Results}

These Class-C accelerometers are of significantly variable accuracy, with that variation sometimes dependent on which axis or individual device is considered (ㅌ) Table $S 1$ available as an electronic supplement to this paper). All brands tested have some sensitivity errors greater than $1 \%$ (up to $2.4 \%$ ) so would need individual box tests and amplitude scaling to bring them within the $1 \%$ ANSS guidance for sensitivity. There are excessively large offsets (more than $\sim 10 \%$ of full scale) in some axes of some models, including some, which likely result in significantly asymmetric clipping, thus, to lowered effective recording range. Orientations relative to sensor cases are within $4^{\circ}$ and the majority within $2^{\circ}$; however, this too would need individual testing (and axis rotation in processing) to bring all within the ANSS guidance of $1 \%$ cross-axis excitation. A $2^{\circ}$ alignment error equates to $3.5 \%$ cross axis; mitigating even this result is the fact that many uses of these data will use random- or greatest-horizontal motions, and therefore are not particularly sensitive to orientations. Further, these devices are likely to be installed by their hosts, who are not experts and may not orient them accurately or communicate their orientation accurately to network operators. (Hutt et al., 2010, imply $0.6^{\circ}$ accuracy by their $-40 \mathrm{~dB}$ cross-axis guidance, but this is commonly allowed to reach $1^{\circ}$, an industry norm.) The active-axis orientation is measured by $0 \mathrm{~Hz}$ outputs in the four box orientations where the nominal active axis is perpendicular to the $g$ vector. We did not dynamically test for cross-axis sensitivity so sources other than die misalignment may be present; based on general experience with MEMS accelerometers, we suspect such sources are small.)

Drift in some exemplars is very large and likely would make impossible integration of those data to retrieve displacement, a critical function for engineering applications. Most of the sensors have very modest drift over time, drift most likely caused by small temperature variations during the tests. Even some Class-A accelerometers have significant temperature sensitivity; ANSS recommends that all accelerometers and recorders, be at least modestly insulated from temperature variations.

\section{TRANSFER-FUNCTION TESTS}

Transfer functions (TFFs, also called response functions) describe amplitude and phase of the sensor output relative to motions input to the sensor. In this case, we did not have adequate control of time for the input signals, so cannot evaluate phase. Most macroscopic (i.e., traditional non-MEMS) and MEMS accelerometers, including all those reported here, are nearly flat from $0 \mathrm{~Hz}$ to near a fairly high-corner frequency (typically $\geq 100 \mathrm{~Hz}$ ). Analog-to-digital converters (ADCs) and processing by recorders typically lead to amplitude roll off at lower corner frequencies, and control that portion of the TFF. In the case of these Class-C sensors, these matters are controlled largely within the sensor itself; the digitized data are transferred to the host computer via a USB connection and can be further modified during sample-rate adjustments as described in the Introduction.

We measured amplitude response using a linear shake table to input sine wave motion at various amplitudes and frequencies (slightly imperfect sines, e.g., solid lines in Fig. 2a), and comparing the output signals with these input reference 

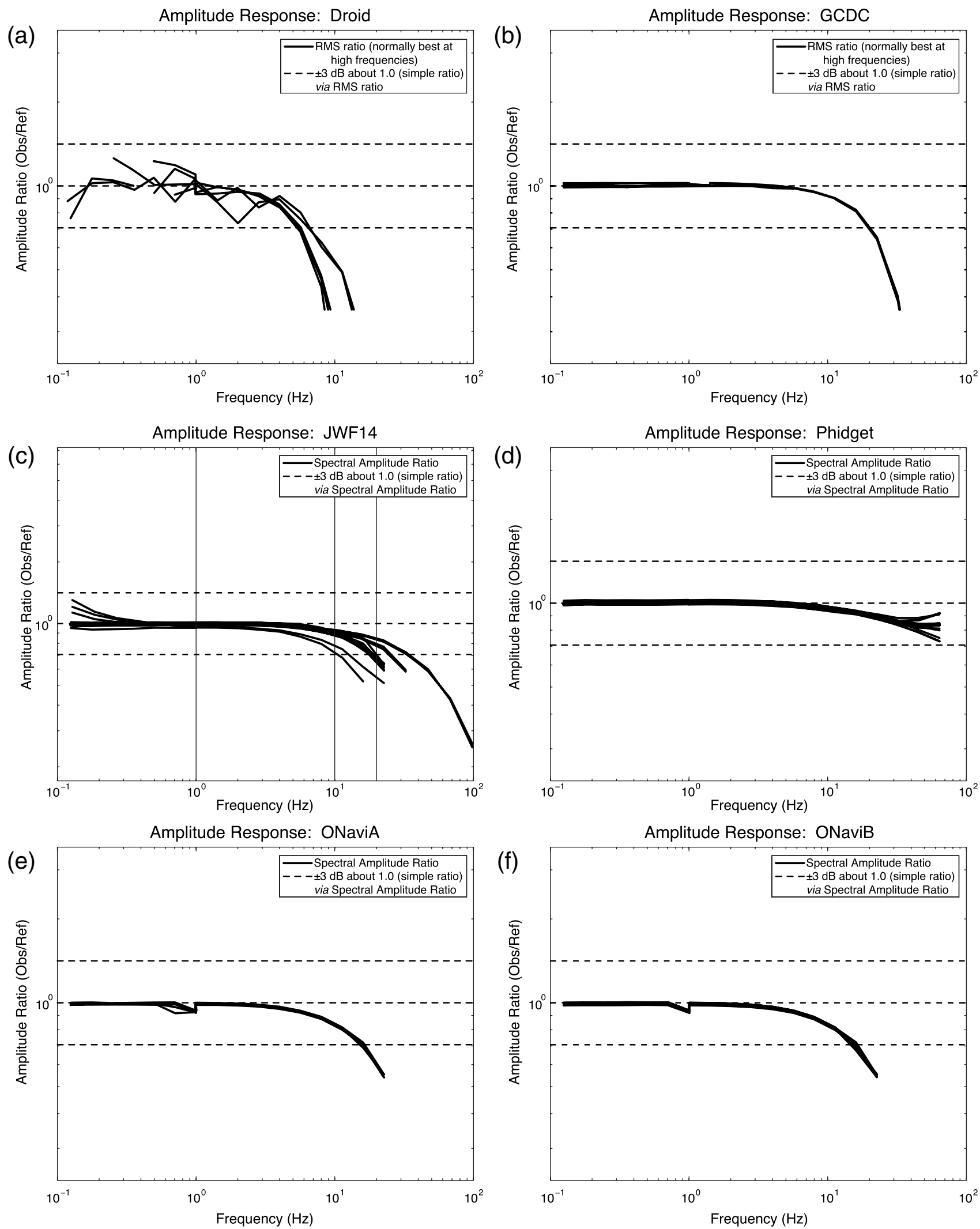

A Figure 3. (a-f) Credible amplitude ratios versus frequency for each model tested. Note varying computational methods. 
signals. In two cases (smart phones and GCDC), sample rates were not stable and accurate, so simple root mean square (rms) amplitude ratios in the time domain had to be used. These rms ratios are quite sensitive to sensor and other noise, particularly for the weak signals required at long periods, which are limited in acceleration by the $40 \mathrm{~cm}$ effective length of the shake table.

In all other cases, a more accurate spectral method was used, comparing the total power in the five frequency bins surrounding the expected (and nearly always peak) sine frequency, as shown in Figure 2b. The use of only the five bins of the peak spectral response greatly improves signal-to-noise ratios by narrowing the bandwidth of the noise; it has shown itself many times to produce credible results over a wider range of frequencies than simple rms (particularly at low frequencies where input signals are limited by the shake-table length) for many types of sensors. Simple rms often fails quite badly, greatly deviating from expected responses where the spectral method does not. An example of our analysis results for a single frequency sine wave is shown in Figure 2; the sine-wave segment is selected by an analyst (the first few seconds of that segment are shown in Fig. 2). In this series of tests, we lacked an independent reference sensor for the shake table so we generated synthetic sine waves of the known input amplitude and frequency, but no phase information, and took amplitude ratios relative to this synthetic sine wave.

\section{Results}

Most of these sensors had to be upsampled, typically from 50 samples/s to the 200 samples/s we normally use for such analyses and a standard in ANSS. Phidgets had to be downsampled slightly, from 250 to 200 samples/s, which we use the MATLAB function resample(), and an integer ratio of upsample, then downsample very close to the ideal, typically parts per billion, and, in this case, exact with a ratio of four up, then five down. The low sample rates of the other sensors likely are

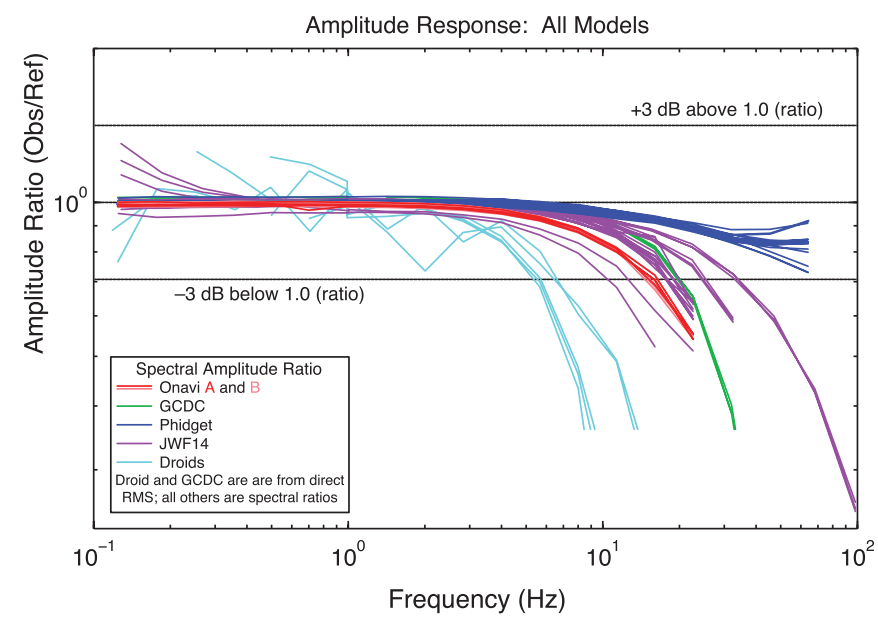

$\Delta$ Figure 4. A summary of amplitude responses for all models, in each case selecting those examples believed to be the most accurate and representative. Colors indicate sensor models (O-Navi A and B are essentially identical, so B is difficult to see). the primary cause of differences in Figures 3 and 4 between the evident corner frequencies.

We also observe soft shouldered behavior below their corner frequencies; this feature is quite visible near $10 \mathrm{~Hz}$. This soft-shouldered feature is equivalent to over-damping an oscillator, and in this instance means that the sensor responses fall below the widely used $-3 \mathrm{~dB}$ in-band limit, well below the nominal corner frequency, at anywhere from about 7 to $80 \mathrm{~Hz}$. Commonly, Class-A instruments are nearly flat to about $80 \%$ of the Nyquist frequency then fall sharply, this largely controlled by the ADC. It is likely that the Class- $\mathrm{C}$ sensors use internal oversampling and digital decimation of some type because MEMS accelerometers are mechanically almost flat in the seismic band, because they have natural frequencies of several hundred hertz or above. However, there is a chance that analog filtering is applied in their feedback loops, and we could not separate these effects. Assuming that ADC digital filtering dominates Class- $\mathrm{C}$ accelerometer responses, their responses are likely something akin to the $\operatorname{sinc}^{n}(x)=[\sin (x) / x]^{n}$ decimation filters, which are common in low-cost delta-sigma ADCs, including those in the GeoSIG/USGS NetQuakes Class-B instrument.

If desired, such softening near the corner frequency can be partially deconvolved to something more like typical Class-A accelerographs with relative ease during processing, a relatively stable partial deconvolution. NetQuakes and all other ANSS data are stored without deconvolutions of any type, but are stored as is, but with TFF specifications in their metadata. This practice leaves any deconvolution under the control of users. Similarly, Class-C data would also be stored unmodified, but with their TFF in the metadata.

The smart phones could not be evaluated for their TFFs except in the most general sense using a simple rms ratio in the time domain; waveforms were peculiar. GCDCs had good waveforms, but because of sample skipping the sample rate was not stable enough for spectral analysis; their simple rms ratios in the time domain yield reasonable results. The other sensors were evaluated with the spectral ratio technique. They all exhibit a more or less flat response at low frequency with one overdamped corner at high frequency. This pattern is in general conformity with Class- $\mathrm{A}$ and Class- $\mathrm{B}$ accelerometers though the sharpness and phase of the high-frequency corner rolloff varies, most often in a manner controlled by the deltasigma ADC decimation filtering. The non-MEMS forcefeedback accelerometers of typical Class-A accelerometers may have natural frequencies near the seismic passband (e.g., $100 \mathrm{~Hz}$ ) and contribute significantly to the overall response.

\section{CLIP AND LINEARITY TESTS}

The degree to which the sensitivity of each accelerometer is constant at a fixed frequency with changes in input acceleration amplitude is called linearity. At some high amplitudes, sensitivity departs from an acceptably linear response by more than about $1 \%$ of its lower-amplitude sensitivity, which is termed as the soft clipping acceleration level. Above a somewhat higher amplitude, the sensor output does not change with 


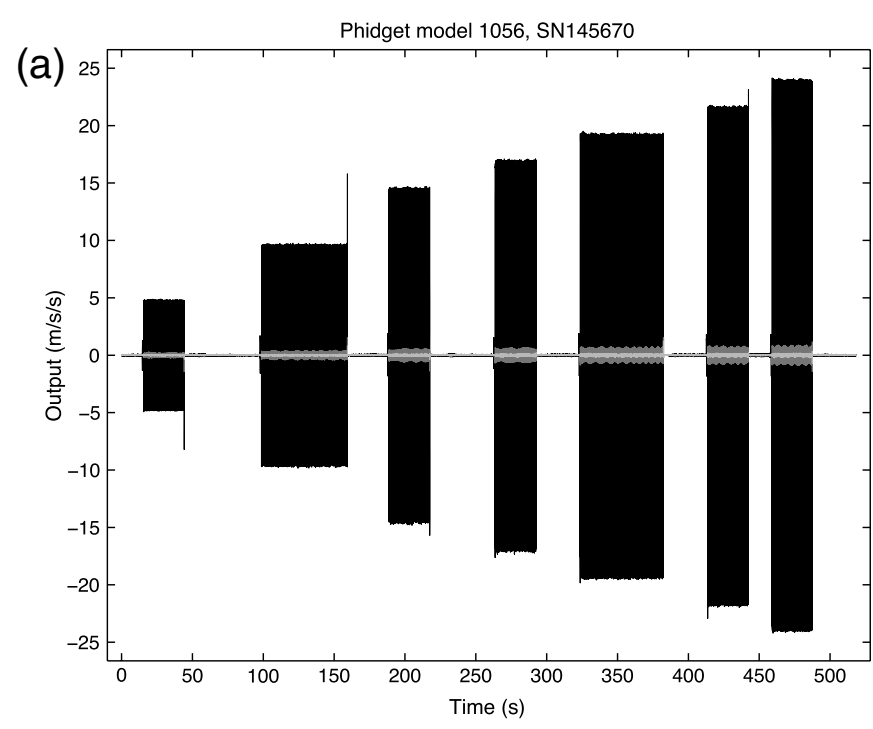

(C) "X; QCN Sensor; ONaviB SN b1"; [upsampled by a factor of 8] (C) Nominal Frequency $=5.6569 \mathrm{~Hz}$; Measured (FFT) Frequency $=5.6536 \mathrm{~Hz}$
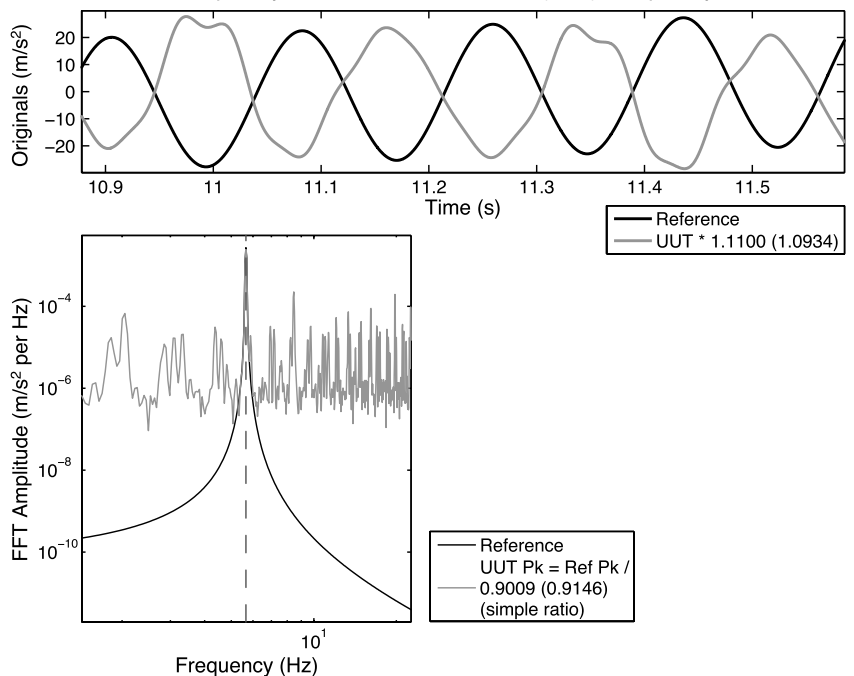

(b)
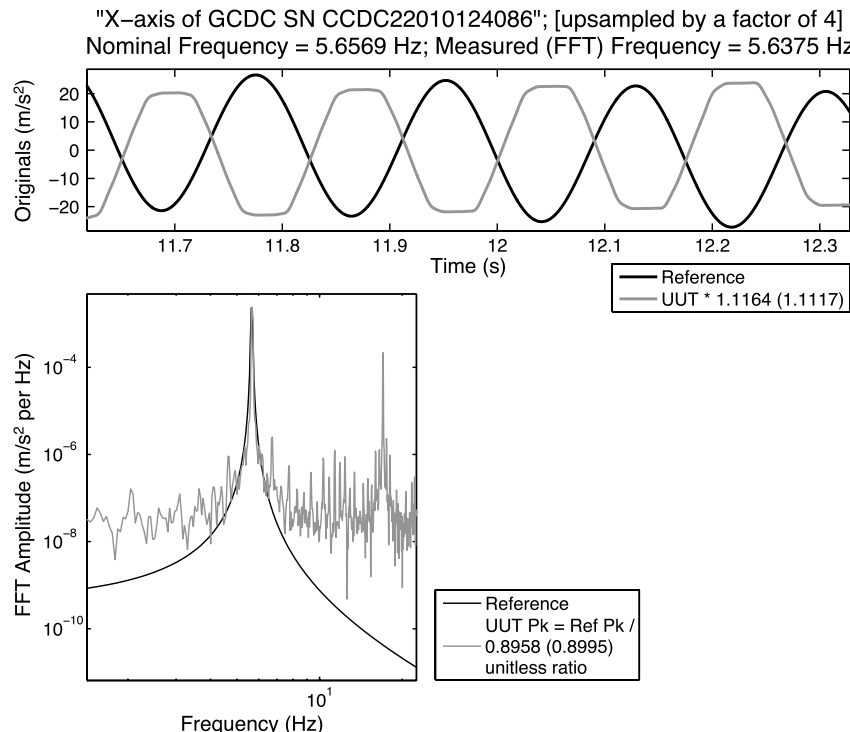

"X, Phidget 1043 prototype, SN 999990"
(d) Nominal Frequency $=5.6569 \mathrm{~Hz}$; Measured (FFT) Frequency $=5.6748 \mathrm{~Hz}$
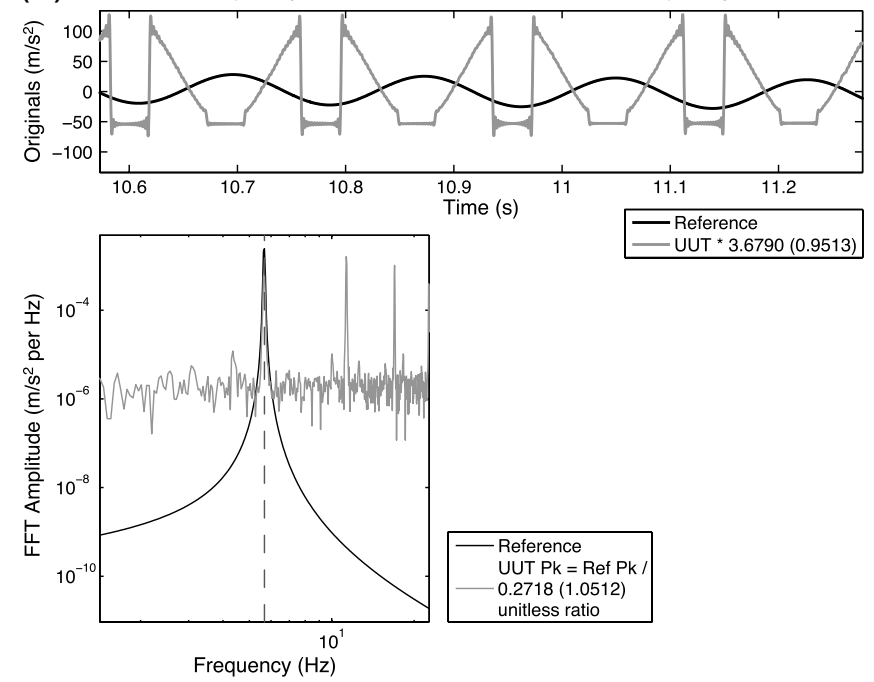

- Figure 5. Examples of time series used for clip/linearity tests: (a) time series of an entire sequence of amplitudes at one frequency (light and dark gray are the off-axis components); (b) an example of typical clipping (gray in upper panel; ignore phase); (c) an example of adequate clipping behavior; and (d) an example of force-to-value clipping, which appears to have been corrected in production versions.

increasing amplitude-the hard clipping amplitude. Normally, soft and hard clipping are very close together and generally not distinguished, but some of these Class-C sensors appear to soft clip significantly for unknown reasons.

The inputs and analysis for clipping and linearity progress much as TFF tests, but the input-sine shaking is held at one or more fixed frequencies while a range of amplitudes is applied (Fig. 5a); in this case, we input as many as ten amplitudes from $0.25 \mathrm{~g}$ to $2.5 \mathrm{~g}$ at a midband frequency $4 \sqrt{ } 2 \mathrm{~Hz}$. For a given frequency, one hopes to see a flat sensitivity response expressed as output/input ratio to within a few percent of the hardclipping amplitude, and then some kind of gentle rolloff in which the output signal becomes a cleanly clipped sine wave. In the time domain, a clipped sine wave for a typical sensor would look normal up to the soft-clip amplitude, then progressively distorted up to the hard-clip amplitude at which it would become a constant until the input signal fell below the clipping amplitudes, there resuming normal appearance. Although the span between soft and hard clipping may be infinitesimal, the amplitude ratio will decrease only slowly because a distorted, then clipped, sine still has a lot of energy at the original sine frequency (Figs. 5 and 6); when clipping, the amplitude ratio decreases because the input amplitude is increasing, whereas the apparent output amplitude stays about the same. Thus, soft clipping may be evident only to the eye in the time domain or if one performed something like a harmonic distortion test. We did not correct for TFF or $0 \mathrm{~Hz}$ sensitivity before performing this test. 
(a)

(c)
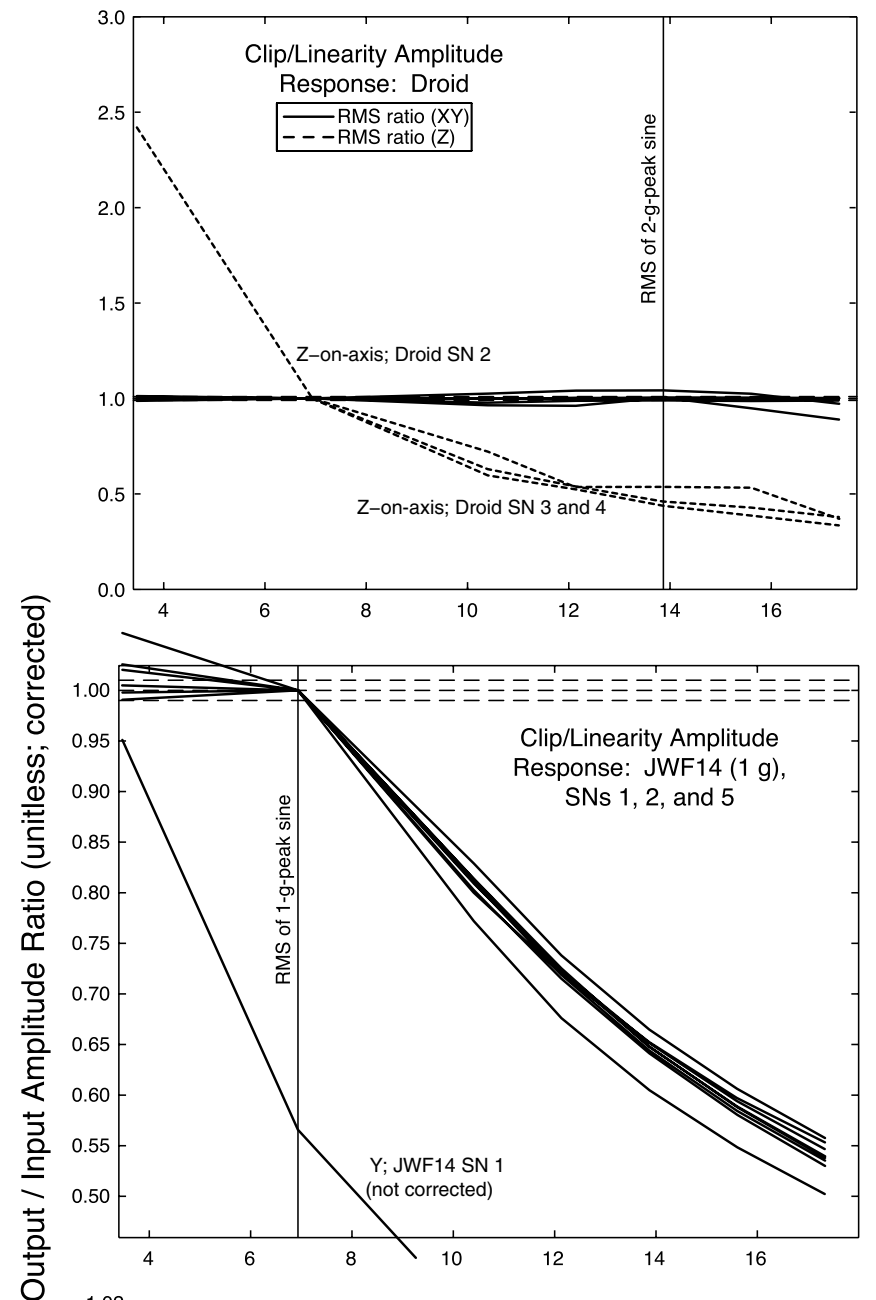

(e)

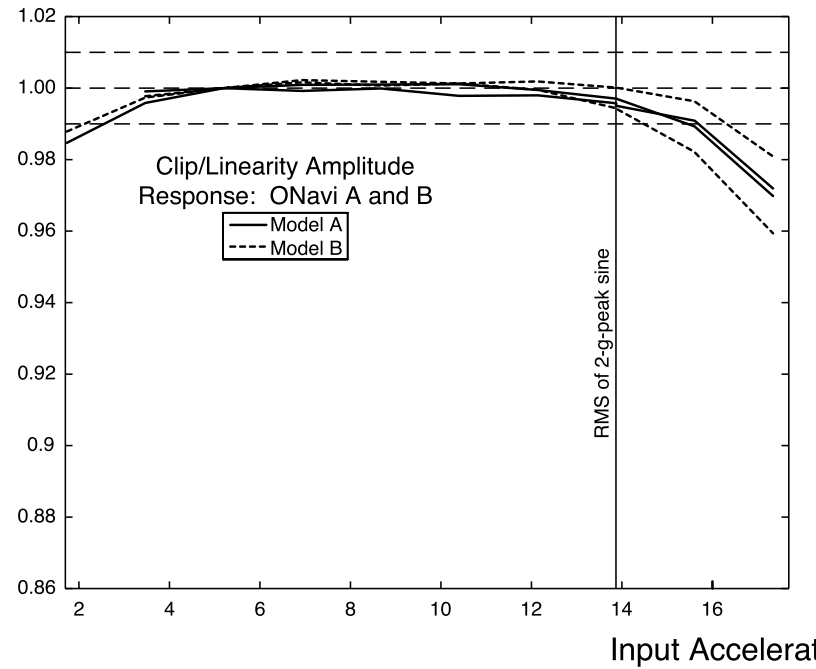

(b)

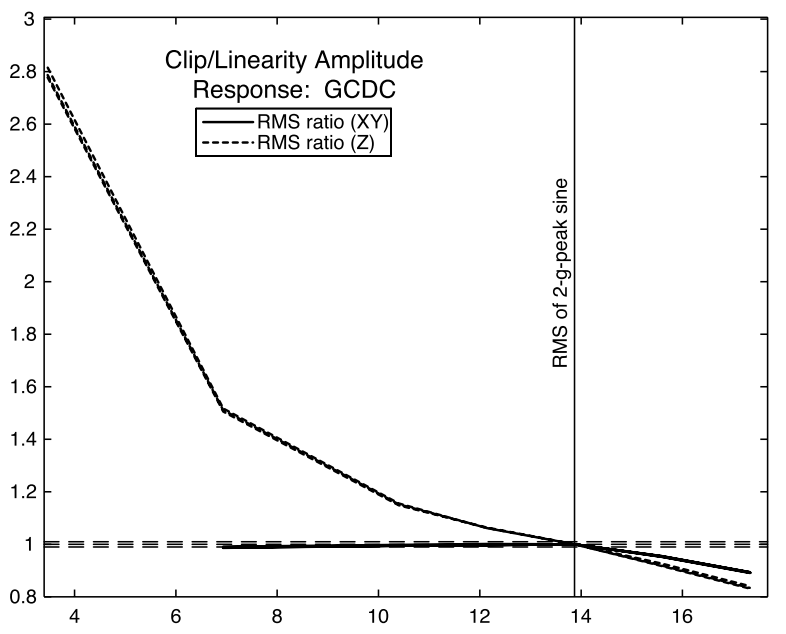

(d)

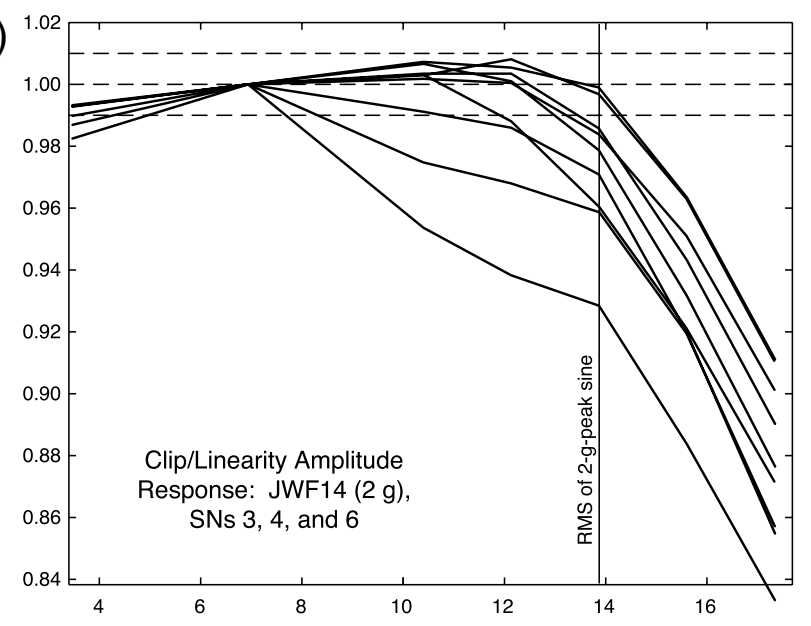

(f)

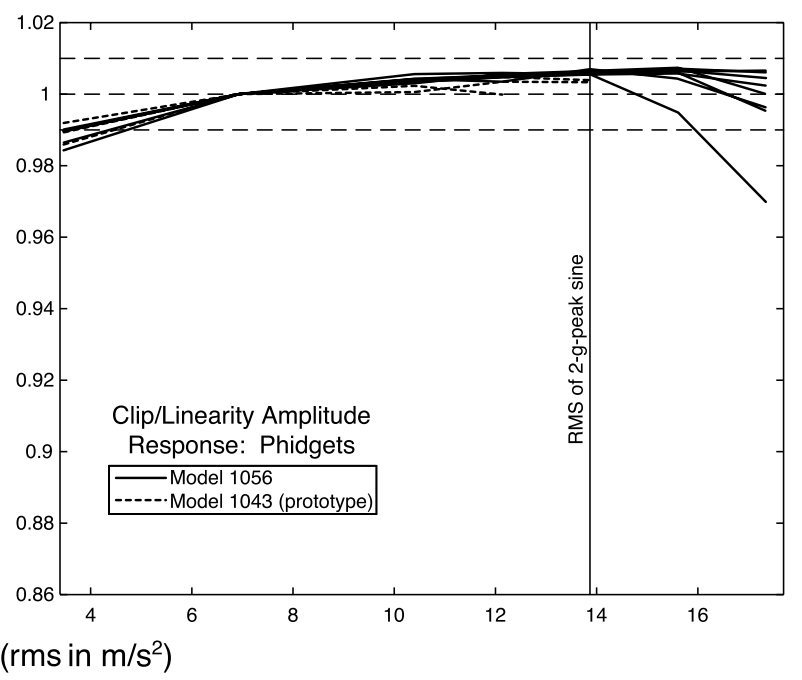

A Figure 6. Summary of clip and linearity testing; spectral amplitude ratio method if not otherwise stated. The dashed horizontal lines show the expected ratio (unity) $\pm 1 \%$ of that ratio (linear scale), the maximum span expected by the ANSS in Class-A and Class-B sensors. Here we have shifted to unity, the amplitude ratio of each sensor at the rms of a $1 \mathrm{~g}$ sine $\left(6.9 \mathrm{~m} / \mathrm{s}^{2}\right.$, except GCDC corrected at rms of $\left.2 \mathrm{~g}\right)$, effectively correcting them for individual sensitivity errors at that amplitude. The vertical solid lines show expected clipping amplitudes; vertical scaling differs between models. Model and axes are indicated by text and line type. 


\section{Results}

Examples of waveforms and analysis when the sensors are driven beyond their hard-clip level are given in Figure 5b-d. They exhibit a reasonably typical clipping pattern (Fig. 5b), a similar pattern distorted by sensor and upsampling filters (Fig. 5c), and the single Phidgets prototype, which, when clipped to a fixed value, causes large excursions from the other amplitude extremes (Fig. 5d). The latter apparently has been changed in production models to the symmetrical clipping familiar in seismology and engineering.

As summarized in Figure 6, most of the sensors clipped in a reasonably well-behaved manner, rolling over above their nominal clipping amplitudes; however, there are a number of exceptions. As mentioned, the single prototype Phidgets (model 1043) clipped atypically (Fig. 5d), though we believe this has been corrected in production versions. The smart phones clipped asymmetrically; the cause is not clear. The JWF14 sensors exhibit two behaviors and variation within each. Most of the outwardly premature clipping is due to three of those six sensors being $\pm 1 g$ devices so that they should clip at low amplitude; the other three are $\pm 2 g$ and behave about as well as the O-Navi and Phidgets sensors. The model 1056 Phidgets and O-Navi A and B behaved well, as did the horizontal axes of the GCDC. The $\pm 2 g$ JWF14, model 1056 Phidgets, and O-Navi A and B sensors all show lower outputs at low frequencies; this result may simply be due to higher signal-to-noise at those frequencies as a result of the smaller inputsine amplitudes. The behavior of $Z$ axes of the GCDC and smart phone sensors may be due to premature clipping caused by in-sensor corrections for the $1 g$ gravitational field normally seen by $Z$ axes.

\section{NOISE TESTS}

The noise generated by the sensor itself is a measure of the smallest signals that can be resolved by that instrument, and along with clipping levels determine its useful operating range

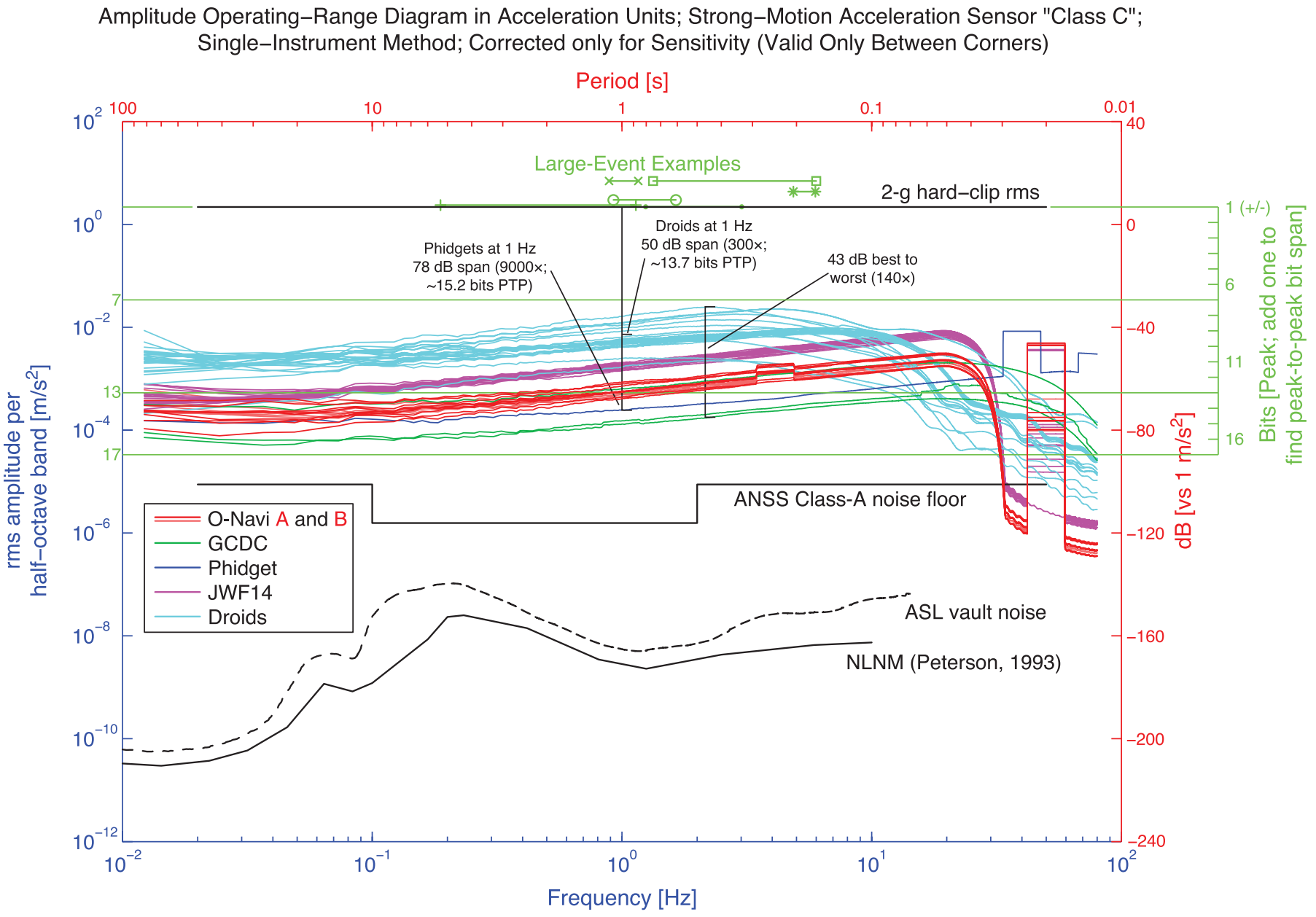

A Figure 7. Operating ranges for the tested sensors range from the noise floor (half-octave total rms) to the clipping level (rms of justclipping $2 g$ sine). Box-like features are caused by integrating to half-octaves narrowband peaks, which are of little impact to sensor performance. All sensors roll off at their anti-alias corners, set by their sample rates; the Phidget has the highest such corner because of its high-sampling rate. Large-Event Examples are at the peak amplitude (times 0.707 for comparison to rms noise) and span the half-width of their spectrum. 
(Holcomb, 1989; Sleeman et al., 2006; Evans et al., 2010, 2012).

Instrument self noise is generally estimated from records recorded, when the sensors are fixed to a low-noise pier during a late-night interval with low cultural and wind noise. In Class$\mathrm{A}$ and Class-B accelerometers, background ambient noise at the test site sometimes can be seen even under these conditions, so a coherency method is used to estimate which part of the noise is inherent to the sensor, while rejecting coherent portions of the noise as likely caused by external seismic or electronic sources. Ambient seismic noise is dominantly from oceanic microseisms and local cultural and weather sources, so is coherent between collocated sensors on the same pier. In the case of these Class- $\mathrm{C}$ sensors, however, the sensor noise is well above the site noise, which made the coherence method and even night recording unnecessary. Instead, all of the sensor output is attributed to instrument noise. We did not correct for TFF or $0 \mathrm{~Hz}$ sensitivity before performing this test; a small amount of the noise variation seen results from variations in $0 \mathrm{~Hz}$ sensitivity, but this is a tiny correction.

\section{Results}

Figure 7, with one line per measured axis, and (E) Table $S 2$ of the electronic supplement summarize our noise results as well as showing a clipping level at the rms of a just-clipping $(2 g$ peak) sine wave. The span between these metrics from rms clip to rms noise is the useful operating range of each sensor. There is a wide range of instrument noise levels among these devices ( $43 \mathrm{~dB} \sim 140 \times$ in amplitude near $1 \mathrm{~Hz}$ ) from the worst (smart phones) to the best (two axes of the single GCDC tested). Phidgets are the next quietest. We obtained only one noise sample, that for a different individual than tested at ASL; that Phidgets has a useful operating range of $78 \mathrm{~dB}$ at $1 \mathrm{~Hz}$. Equivalent bits resolution (peak only, i.e., one sided and one-bit less than peak-to-peak resolution) are shown at the right margin of the figure and range from about 8 to 16 useful bits $(9-17$ bits peak-to-peak). () The summary in Table $\mathrm{S} 2$ shows equivalent bits resolution for the full peak-to-peak range.

\section{DOUBLE-INTEGRATION TESTS}

Attempting to recover transient or permanent displacement signals from acceleration records is a highly sensitive, but nonspecific, test of end-to-end system viability for strong-motion work. This is an important test because engineers and many seismologists must use acceleration records to recover permanent displacements (e.g., near-field fault fling and interstory drift, permanent structural deformation; Çelebi et al., 2004; Çelebi, 2008; Chopra, 2012). The test is highly sensitive because even very tiny acceleration steps, changes in trend, missing samples, hysteresis, and other problems are greatly amplified by the integration process into large signals that are easily identified; in some cases, these errors in displacement are comparable to or larger than the input signal. Long-period wander in the displacement traces is normal in our experience (Classes A and B), because of imperfections in temperature corrections and the baseline-corrections we apply. It is the amplitudes and waveforms of the input steps that need to be recovered accurately.

We use the same linear shake table as for TFF and clip/ linearity tests, but input a rounded square wave in displacement (400 $\mathrm{mm}$ peak to peak; peak accelerations $\sim 0.5 g$; peak velocities $\sim 800 \mathrm{~mm} / \mathrm{s}$; period $11.6 \mathrm{~s}$; and a stationary waiting period at each end of the shake table of $5 \mathrm{~s}$ ). Each excursion from one end of the table to the other (transit duration $\sim 0.77 \mathrm{~s}$ ) amounts to a quasi-permanent displacement offset of the kind that must be recovered reliably.

Recovering displacement from acceleration is formally an underdetermined problem (zero sensor output at $0 \mathrm{~Hz}$ ), which has led to many methods for preprocessing the acceleration records to remove or mitigate artifacts due to fixed or drifting offsets, for example. Commonly, the analyst removes means or trends from the acceleration record, applies a low-cut filter, then performs a numerical integration twice. This method works well for records, which have no permanent displacements. However, we desire to recover permanent displacements and cannot use a low-cut filter; we fit a line to the result of the first integration (velocity), correct the acceleration accordingly, and then double integrate the corrected acceleration to displacement. We did not correct for TFF or $0 \mathrm{~Hz}$ sensitivity before performing this test, but the signals are largely unaffected because these are midband signals other than during the $5 \mathrm{~s}$ motionless periods; amplitudes will vary some, but waveforms should be a good match.

\section{Results}

The results of our double-integration tests are shown in Figure $8 \mathrm{a}-\mathrm{f}$, each labeled by sensor model. Several sensors perform poorly in this test (Figs. 8a-c) in the sense of not recovering waveforms well or having large extraneous excursions in displacement; these sensors include the smart phones, JWF14, and two of six axes of the GCDC (the latter may be caused by missing samples during peak accelerations). Smart phone and GCDC sampling problems are also clear in the uneven step durations most evident in acceleration traces; they should overlap very closely, as do the other sensors. Hysteresis is easily seen in the velocity traces of all models, but particularly in the smart phones, JWF14, and GCDC (the same two traces mentioned). The O-Navi A and B have noticeable hysteresis that does not seem to distort the displacement result, though there are visible second-order changes in trend at a number of larger hysteretic steps. The Phidgets have slight drift, but no evident hysteresis.

Long-period wandering of the displacement baselines is typical of these tests using our simple processing method, which does not properly account for exponential accelerationbaseline drift due to temperature variations and perhaps other sources. The remaining drift amplifies into the displacement wander seen, but is of no importance here and is seen routinely in Class A and Class B sensors tested this way. Earthquake records would be similarly affected by temperature etc., however, for such valuable records, custom, if demanding baseline 
(a)
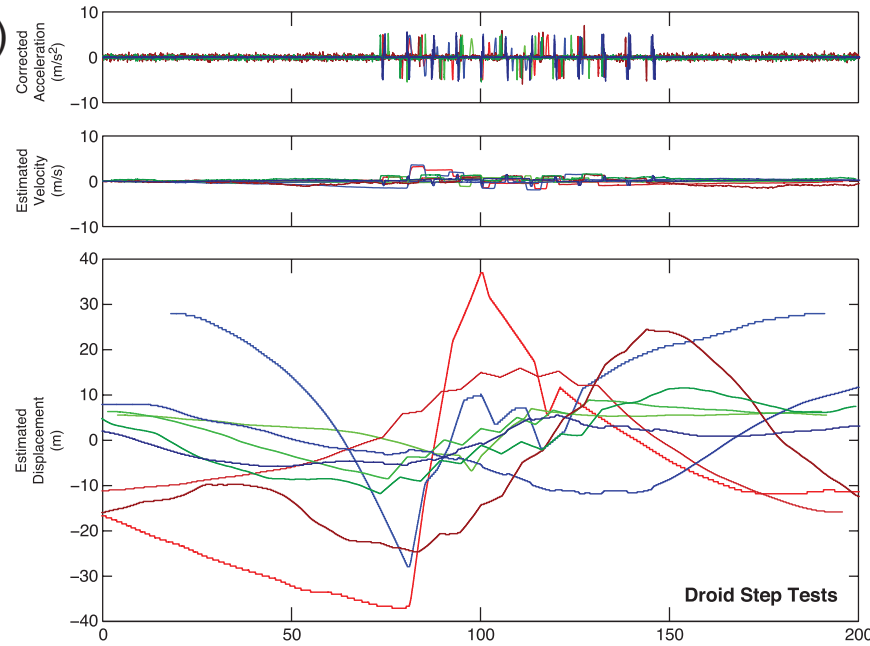

(c)

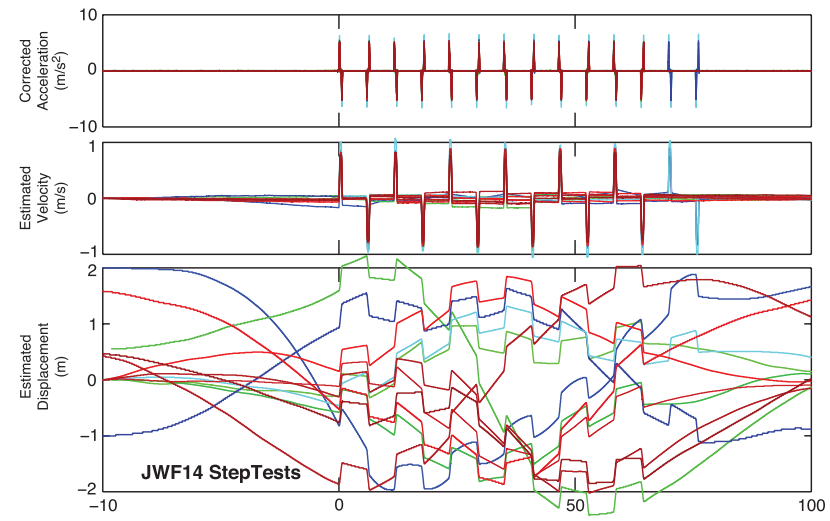

(e)

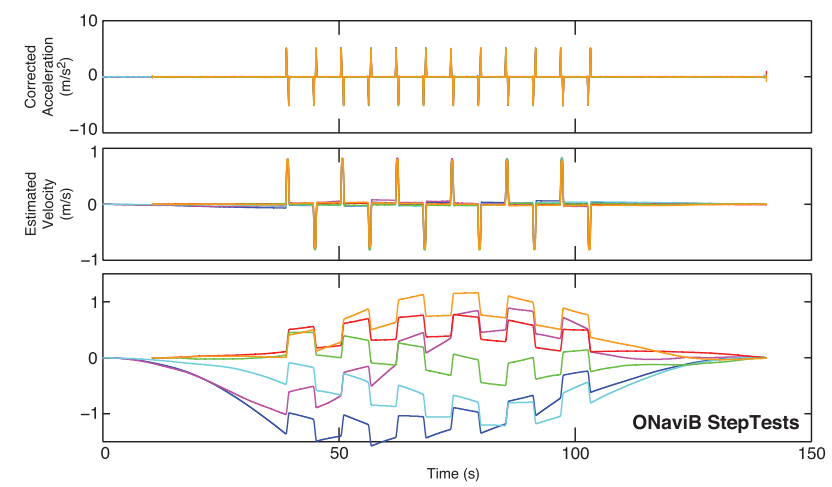

(b)

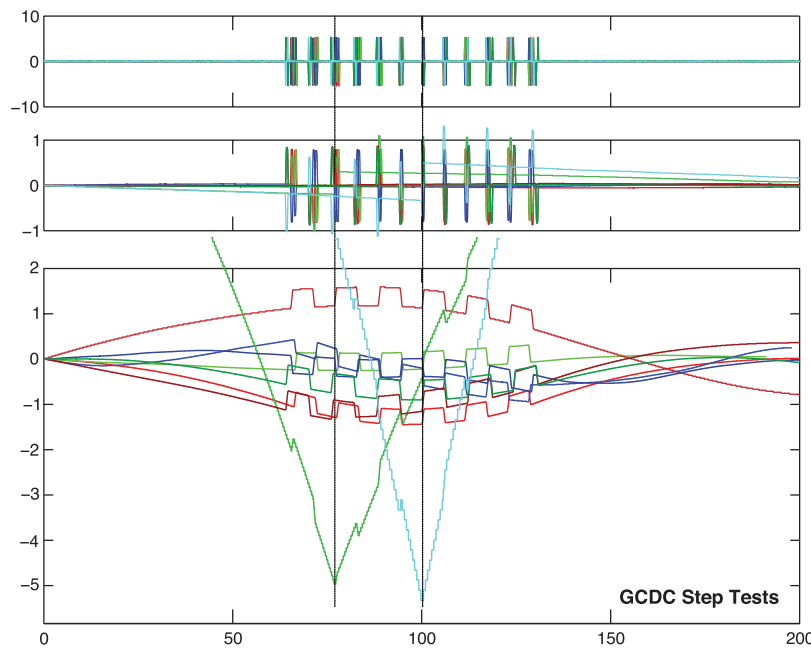

(d)

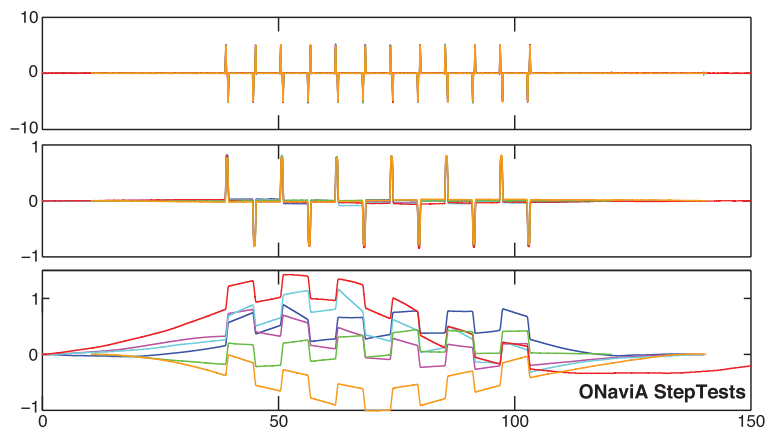

(f)
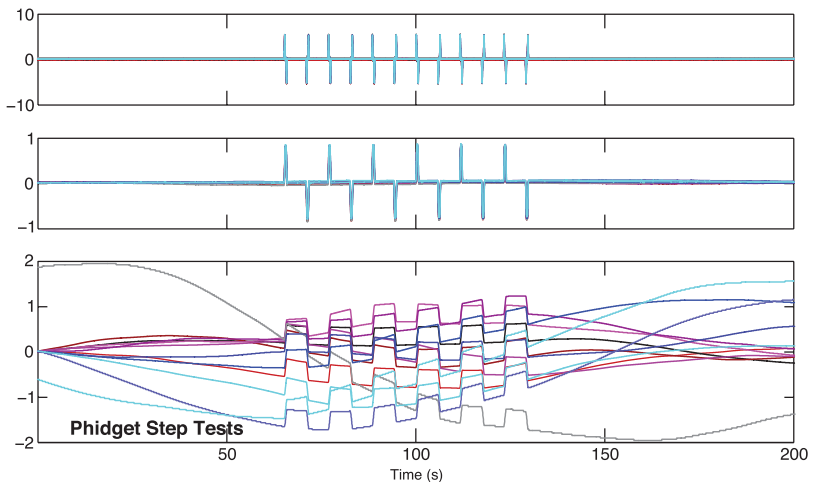

(g)

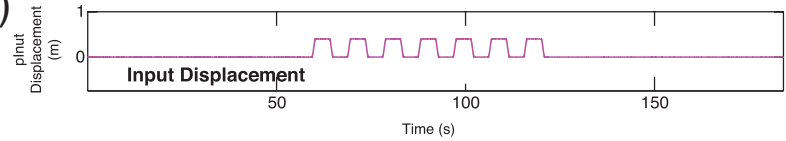

- Figure 8. Double integration results for all tested axes. Figures (a-f) are labeled by sensor model; all traces evaluated for a given model are shown in that figure and can be distinguished by color (which has no other meaning). Panel $(\mathrm{g})$ is the approximate input displacement waveform. Within each figure, the corrected acceleration traces and resulting velocity and displacement estimates are shown; in (b) the large green and blue chevrons are large displacements clipped to allow plotting. Displacement reveals overall performance, whereas velocity shows hysteresis most clearly and acceleration shows the best sample-rate stability.

corrections generally allow one to recover displacements better than the automated method used for Fig. 8.

Step amplitudes and waveforms seem reasonably good for the O-Navi A and B and the Phidgets. The GCDC has reason- able amplitude and waveform, but not duration because of sample-rate instability, except in the two that seem to be missing a critical sample during high-acceleration intervals. The smart phones sensors perform very poorly in waveform, 
whereas JWF14 sensors intermittently recover reasonable amplitudes and some semblance of waveforms, but are otherwise quite poor.

\section{CONCLUSIONS}

(E) Table S3 summarizes the performance of sensors in each test with a mix of objective and interpretive rankings among the five models tested, as well as a summary ranking. The sensors exhibit a very wide range of performance: three are presently unacceptable per general ANSS guidance; two are acceptable as is, both O-Navis (though sample rates need to be higher than are presently provided by QCN) and the Phidgets. The O-Navi sensors have less than ideal offsets. Overall, the Phidgets may have the edge in performance because of smaller offsets, lower hysteresis, and higher currently available sample rates.

If operated at relatively high sample rates (100-200 samples/s), either O-Navi or Phidgets sensors should produce data of use to ANSS, if those data can be properly tagged as Class- $\mathrm{C}$ to ensure that their uses are appropriate. As mentioned above, analog instruments of significantly lower resolution were used for some decades and produced much useful information. Their data found many applications, including reliable pictures of regional seismicity and strong shaking, and several generations of earthquake-safety building codes.

There are a number of potential paths to create a Class-C instrument compatible with current ANSS operations, in particular with the ANSS Earthworm software system, for capture and analysis of seismic data. There are at least two such paths in development and likely others we do not know of; in any case the details of those implementations will change over time so are not listed here. (E) Table S4 is a list of parts that could be used in a hypothetical high-end Class-C DAS, simply as an example of the available options and likely cost ranges.

In all, these results are hopeful, suggesting that it may be possible to integrate some Class-C instruments into ANSS operations to produce information useful to ShakeMap (Wald et al., 1999), seismological research, and earthquake engineering. $\mathbf{S}$

\section{ACKNOWLEDGMENTS}

The Community Seismic Network (CSN; R. Guy) is funded by Gordon and Betty Moore Foundation and NSF award CNS 0932392; special thanks to Leif Strand of CSN for authoring the Phidgets testing application(s). The Quake Catcher Network (QCN; E. Cochran, J. Lawrence, and A. Chung) is a joint project of the USGS and Stanford University. MyShake (Droid smart phones; R. Allen) is funded by Deutsche Telekom.

A note on units: In this report the unit " $g$ " (in italics) is used to mean the acceleration of gravity at the Earth's surface; it does not mean grams ( $" g$ " not italicized). One "standard $g$ " is $9.80665 \mathrm{~m} / \mathrm{s}^{2}$, but at the site of these experiments (Albuquerque Seismological Laboratory) the local value of $1 g$ is
$9.79188087 \mathrm{~m} / \mathrm{s}^{2}$. We correct all our results from the local value of $g$ to the standard value.

A note on trademarks: Trademarked or otherwise restricted names used in this report include Droid, Google Nexus One, HTC Magic, iPhone, O-Navi LLC, Phidgets Inc., JoyWarrior, and Code Mercenaries Hard- und Software GmbH, Gulf Coast Data Concepts (GCDC), Excel, GeoSIG, Kinemetrics SMA-1, and MATLAB. Any use of trade, firm, or product names is for descriptive purposes only and does not imply endorsement by the U.S. Government.

\section{REFERENCES}

ANSS Technical Integration Committee (TIC) (2002). Technical guidelines for the implementation of the Advanced National Seismic System, version 1.0, U.S. Geol. Surv. Open-File Rept. 02-9296.

ANSS Working Group on Instrumentation, Siting, Installation, and Site Metadata of the Advanced National Seismic System Technical Integration Committee (2008). Instrumentation Guidelines for the Advanced National Seismic System, U.S. Geol. Surv. Open-File Rept. 2008-1262, 41 pp.

Çelebi, M. (2008). Real-time monitoring of drift for occupancy resumption, Proc. 14th World Conference on Earthquake Engineering (14WCEE), Beijing, 12-17 October 2008.

Çelebi, M., A. Sanli, M. Sinclair, S. Gallant, and D. Radulescu (2004). Real-Time seismic monitoring needs of a building owner-and the solution: A cooperative effort, Earthq. Spectra 20, 333-346.

Chopra, A. K. (2012). Dynamics of Structures, 4th Ed., Prentice-Hall, Upper Saddle River, New Jersey, International Series in Civil Engineering and Engineering Mechanics, 992 pp.

Cochran, E. S., J. F. Lawrence, C. Christensen, and R. Jakka (2009). The Quake-Catcher Network: Citizen science expanding seismic horizons, Seismol. Res. Lett. 80, 26-30.

Evans, J. R., F. Followill, C. R. Hutt, R. P. Kromer, R. L. Nigbor, A. T. Ringler, J. M. Steim, and E. Wielandt (2010). Method for calculating self-noise spectra and operating ranges for seismographic inertial sensors and recorders, Seismol. Res. Lett. 81, 640-645, doi: 10.1785/ gssrl.81.4.640.

Evans, J. R., F. Followill, C. R. Hutt, R. P. Kromer, R. L. Nigbor, A. T. Ringler, J. M. Steim, and E. Wielandt (2012). Method for calculating self-noise spectra and operating ranges for seismographic inertial sensors and recorders, Erratum, Seismol. Res. Lett. 83, 586, doi: 10.1785/gssrl.83.3.588.

Holcomb, L. G. (1989). A direct method for calculating instrument noise levels in side-by-side seismometer evaluations, U.S. Geol. Surv. OpenFile Rept. 89-214, 35 pp.

Hutt, C. R., J. R. Evans, F. Followill, R. L. Nigbor, and E. Wielandt (2010). Guidelines for standardized testing of broadband seismometers and accelerometers, U.S. Geol. Surv. Open-File Rept. 20091295, $62 \mathrm{pp}$.

Hutt, C. R., J. Peterson, L. Gee, J. Derr, A. Ringler, and D. Wilson (2011). Albuquerque Seismological Laboratory-50 years of global seismology, U.S. Geol. Surv. Fact Sheet 2011-3065, 4 pp.

Luetgert, J. H., J. R. Evans, J. Hamilton, C. R. Hutt, E. G. Jensen, and D. H. Oppenheimer (2009). NetQuakes-A new approach to urban strong-motion seismology, $A G U$ Fall Meeting S11B-1707.

Luetgert, J. H., J Hamilton, and D. H. Oppenheimer (2010). The NetQuakes Project-Research-quality Seismic Data Transmitted via the Internet from Citizen-hosted Instruments, $A G U$ Fall Meeting S51E-03.

Sleeman, R., A. van Wettum, and J. Trampert (2006). Three-channel correlation analysis: A new technique to measure instrumental noise of digitizers and seismic sensors, Bull. Seismol. Soc. Am. 96, 258271, doi: $10.1785 / 0120050032$. 
Wald, D. J., V. Quitoriano, T. H. Heaton, H. Kanamori, C. W. Scrivner, and C. B. Worden (1999). TriNet "ShakeMaps": Rapid generation of instrumental ground motion and intensity maps for earthquakes in southern California, Earthq. Spectra 15, 537-556.

J. R. Evans Earthquake Hazards Science Center U.S. Geological Survey 400 Natural Bridges Drive Santa Cruz, California 95060 U.S.A. jrevans@usgs.gov

R. M. Allen M. Hellweg Department of Earth and Planetary Sciences University of California Berkeley Berkeley, California 94720-4760 U.S.A.
A. I. Chung

J. F. Lawrence Department of Geophysics Stanford University Stanford, California, U.S.A. 94305-2215

E. S. Cochran Earthquake Hazards Science Center U.S. Geological Survey 525 South Wilson Avenue Pasadena, California 91106 U.S.A.

R. Guy Seismological Laboratory California Institute of Technology 1200 East California Boulevard Pasadena, California 91125 U.S.A. 